\title{
Patients' and Clinicians' Preferences on Outcomes and Medication Attributes for Type 2 Diabetes: a Mixed-Methods Study
}

\author{
Thomas Karagiannis, MD, MSc, $P h D^{7}$ (D) loannis Avgerinos, MD, MSc ${ }^{7}$, \\ Maria Toumpalidou, BSc ${ }^{7}$, Aris Liakos, MD, MSc, $P h D^{7}$, \\ Konstantinos Kitsios, MD, MSc, PhD², Georgios Dimitriadis, MD, DPhil ${ }^{3}$, \\ Nikolaos Papanas, $M D, P h D^{4}$, Alexandra Bargiota, $M D, P h D^{5}$, lakovos Avramidis, $M D^{6}$, \\ Anastasia Katsoula, MD, MSc ${ }^{7}$, Anastasios Tentolouris, MD, MSc ${ }^{7}$, \\ Thekla Chatziadamidou, $M D, P h D^{7}$, Stathis Giannakopoulos, $M D, P h D^{7}$, \\ Stavros Alexiadis, $M D^{7}$, Kalliopi Kotsa, MD, $P h D^{8}$, Apostolos Tsapas, MD, MSc, PhD ${ }^{1,2,9}$, \\ and Eleni Bekiari, MD, MSc, PhD ${ }^{1,2}$
}

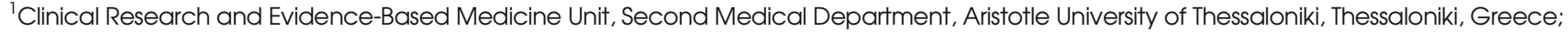
${ }^{2}$ Diabetes Center, Second Medical Department, Aristotle University of Thessaloniki, Thessaloniki, Greece; ${ }^{3}$ Research Institute and Diabetes Center, Second Department of Internal Medicine, National and Kapodistrian University of Athens, Athens, Greece; ${ }^{4}$ Diabetes Center, Second Department of Internal Medicine, Democritus University of Thrace, Alexandroupolis, Greece; ${ }^{5}$ Department of Endocrinology and Metabolic Diseases, University of Thessaly, Larissa, Greece; ${ }^{6}$ First Medical Department, G. Papanikolaou General Hospital, Thessaloniki, Greece; ${ }^{7}$ First Department of Propaedeutic Internal Medicine, National and Kapodistrian University of Athens, Medical School, Athens, Greece; ${ }^{8}$ First Department of Internal Medicine, Aristotle University of Thessaloniki, Thessaloniki, Greece; ${ }^{9}$ Harris Manchester College, University of Oxford, Oxford, UK.
\end{abstract}

BACKGROUND: Patients' views on the relative importance of treatment outcomes and medication attributes for type 2 diabetes may differ from clinicians' perceptions. OBJECTIVE: To assess which treatment outcomes and medication attributes are considered important by patients and clinicians for therapeutic decisions in type 2 diabetes.

DESIGN: Exploratory, sequential, mixed-methods design comprising a qualitative (focus groups) and a quantitative (survey) phase.

PARTICIPANTS: Patients in the focus groups $(n=33)$ and the survey study $(n=656)$ were recruited from 4 and 9 diabetes clinics across Greece, respectively. Clinicians in the survey study $(n=363)$ were identified from Greek registries for healthcare professionals.

MEASUREMENTS: We conducted 6 focus groups to obtain patients' views regarding the impact of type 2 diabetes on their lives. Identified themes informed the development of a survey, which aimed to assess which outcomes and medication attributes are considered most important by patients and clinicians. We calculated odds ratios to compare patients' and clinicians' responses.

RESULTS:The focus groups identified 6 main themes and 15 subthemes. In the survey study, patients

$\overline{\text { Prior Presentation Part of this study was presented at the 54th Annual }}$ Meeting of the European Association for the Study of Diabetes (EASD), 1-5 October 2018, Berlin, Germany.

Electronic supplementary material The online version of this article (https://doi.org/10.1007/s11606-019-05608-0) contains supplementary material, which is available to authorized users.

Received July 4, 2019

Accepted December 6, 2019 were more likely than clinicians to rate prevention of amputation (odds ratio, 9.32; 95\% CI, 6.51 to 13.35), diabetic eye disease $(6.16 ; 4.63$ to 8.21$)$, sexual dysfunction, and stroke as important, while clinicians were more likely than patients to choose risk for hypoglycemia, and reduction of all-cause mortality, HbAlc, and body weight. Compared with clinicians, patients were less concerned about drug cost $(0.16$; 0.11 to 0.23$)$, but more concerned about route of administration and need for less frequent glucose self-monitoring.

CONCLUSIONS: Patients and clinicians differ in the perception of the relative importance of treatment outcomes and drug characteristics. Individual patient preferences should be explored and implemented in the therapeutic decision-making for type 2 diabetes.

KEY WORDS: Type 2 diabetes; Patient preferences; Mixed-methods research; Focus groups; Survey.

$\mathrm{J}$ Gen Intern Med

DOI: $10.1007 / \mathrm{s} 11606-019-05608-0$

(c) Society of General Internal Medicine 2020

\section{INTRODUCTION}

Type 2 diabetes mellitus (T2DM) is a chronic disease in which self-management and patient participation can play an important role in achieving glycemic control or preventing longterm complications. ${ }^{1,2}$ Moreover, patients have to live with the consequences of these treatment decisions; hence, it is important to engage their preferences into clinical practice 
and incorporate their active participation in therapeutic decision-making. ${ }^{3}$ This patient-centered approach can increase the quality of healthcare provision, by improving treatment outcomes and medication adherence, while reducing healthcare costs at the same time. ${ }^{4-6}$ Patient-centered care is a core principle of evidence-based medicine practice ${ }^{7}$ and has been endorsed by the American Diabetes Association (ADA) and the European Association for the Study of Diabetes (EASD) in their joint recommendations for managing T2DM. ${ }^{8,9}$

Studies exploring patient preferences for the treatment of T2DM have used different methodologies, including discretechoice experiments, standard gamble, survey questions, or qualitative research. ${ }^{10,11}$ Most available studies have assessed only a limited number of medication attributes or focused on comparisons between specific medications or drug classes. ${ }^{10}$ Moreover, only a limited number of studies have assessed clinicians' preferences in relation to those of patients, even though patients and clinicians may differ in their perceptions of the relative importance of treatment outcomes and medication attributes. ${ }^{12,13}$ In addition, very few studies have incorporated both qualitative and quantitative methods in their design and implementation. Mixed-methods research has been advocated as being particularly useful for chronic conditions, because it allows both for in-depth evaluation of peoples' views and experiences regarding an observed effect (via qualitative methods) and for estimation of the magnitude of this effect (via quantitative methods). ${ }^{14,15}$

We sought to explore both patients' and clinicians' preferences when making decisions about the pharmaceutical management of T2DM, by conducting a 2-phase mixed-methods study. In the qualitative phase, we conducted focus groups with patients with T2DM to explore their views, experiences, and concerns regarding management of the disease and its impact on their lives. The findings from the focus group study informed the subsequent quantitative phase, a cross-sectional survey aiming to assess which treatment outcomes and medication attributes are considered most important in a larger sample of patients with T2DM and clinicians.

\section{METHODS}

\section{Study Overview}

We used an exploratory, sequential, mixed-methods design comprising a qualitative (focus groups) and a quantitative (survey) phase. ${ }^{16}$ Integration of qualitative and quantitative data was done through the building approach; items for inclusion in the survey were built upon themes and subthemes identified through the focus groups, while patients' phrases informed the wording of survey questions. ${ }^{16}$ We report the 2phase mixed-methods study in accordance with pertinent reporting guidance ${ }^{17,18}$ and adopting a contiguous approach by presenting qualitative and quantitative findings separately. ${ }^{16}$

\section{Focus Group Participants}

In contrast to one-to-one interviewing, which aims to obtain individual beliefs and attitudes, a focus group has a social orientation which encourages the interaction between participants who share certain common characteristics or experiences. ${ }^{19}$ Therefore, the choice of focus groups over personal interviews was based on our expectation that patients with T2DM would be interested in each other's views and experiences related to the disease, allowing them to explore and clarify issues of importance to them in ways that would be less accessible in individual interviews. This advantage of social interaction has been evident in previous focus group studies in patients with T2DM. ${ }^{20-22}$

We used purposive sampling methods ${ }^{23}$ to identify patients with T2DM who were willing to participate in the focus groups, after having been informed about the study's aims and procedures. Patients were identified from 4 diabetes outpatient clinics, provided they were able to understand and speak Greek at a level necessary to participate in a discussion. Each focus group size was planned to range between 6 and 12 participants. ${ }^{24}$ We conducted separate focus group sessions based on the duration of T2DM (using 10 years since diagnosis as a threshold), on the rationale that patients' experiences and perspectives might differ based on the time since diagnosis. For example, patients who have had T2DM for many years often require a combination of various antidiabetic medications, including injectable drugs, and are more likely to have experienced disease-related complications, ${ }^{25}$ as opposed to those who have been recently diagnosed. All participants signed an informed consent form before taking part in a focus group session.

\section{Qualitative Data Collection}

The focus group sessions were facilitated by a psychologist with prior experience in moderating patient group discussions (M.T.), who introduced the topics under discussion, asked questions, and ensured that all participants had the opportunity to contribute in the conversation. A second researcher (T.K.) observed all sessions and was responsible for audio recording, taking field notes and helping the facilitator keep to times. Discussions were based on a semi structured topic guide (Appendix Text Box 1) which was developed a priori by consensus between 4 researchers (T.K., M.T., E.B. and A.T.). Audio data were transcribed verbatim prior to main analysis; however, a preliminary qualitative data analysis was done simultaneously with the data collection process with the aim of achieving data saturation. ${ }^{26}$

\section{Qualitative Data Analysis}

We undertook a thematic analysis ${ }^{27}$ under the general principles of grounded theory, ${ }^{28}$ in the sense that data analysis of the focus groups involved generation of themes as an ongoing iterative process. The analysis comprised 4 main steps: (1) 
preliminary coding and familiarization with the field notes data; (2) application of the coding scheme after reading the transcripts several times and highlighting codes, defined as salient or frequently mentioned words or phrases; (3) categorization of codes and quoted text into themes and subthemes; (4) identification of themes and subthemes that were relevant to the aims of the mixed-methods study. These steps were conducted independently by 2 researchers (T.K. and M.T.). Final interpretation of data was done in consensus between 4 researchers (T.K., M.T., A.T., and E.B.) and was used to inform the development of the survey.

\section{Survey Content}

We designed one patient and one clinician version of a survey to elicit their perceptions of the relative importance of various treatment outcomes and medication attributes for T2DM. Four researchers (T.K., A.T., A.L., and E.B.) were involved in the survey development. Finalization of survey content was done after pilot testing all items on a small sample of patients and clinicians. Based on feedback from pilot testing, the patient survey was refined to appear more user-friendly, by simplifying its language, increasing font size, and adding colored font and a logo at the introductory text. The survey included 3 main questions and few demographic characteristics. The 3 questions of the patient and clinician survey (translated in English) are presented in the Text Box 1 and Appendix Text Box 2, respectively. The patient survey was administered in paper form, while the clinician version was distributed electronically via a centralized data capture system (REDCap, Vanderbilt University, available at https://redcap.med.auth.gr). ${ }^{29}$ All survey data were captured anonymously.

\section{Survey Sample}

Sample size calculation for patients was based on a population size of 770,000 patients with T2DM. ${ }^{30}$ Sample size calculation for clinicians was based on epidemiological data from the Hellenic Statistical Authority, according to which, in 2016, there were approximately 4500 internists, 2800 general practitioners, and 600 endocrinologists in Greece. ${ }^{31}$ Of these, we assumed that $20 \%$ (1600) were active (not retired) clinicians who manage care of patients with T2DM at an outpatient level. Based on these population sizes and using a margin of error of $5 \%$ at a $95 \%$ confidence level, the sample should consist of at least 384 patients and 310 clinicians. We recruited patients from 3 primary care and 6 secondary care practices across Greece that provided care to patients with T2DM. All practices were in urban areas, except for 1 practice located in a rural area. Seven practices were diabetes outpatient clinics coordinated either by internists (6 practices) or endocrinologists (1 practice), while the remaining 2 were general medicine practices. Of note, in Greece, internists are considered providers of both primary and secondary care.

In each clinic, one healthcare provider was responsible for administering the survey and, if necessary, provided minimal assistance to patients to complete it before or after their scheduled encounter with their physician. Following the end of the recruitment period, all completed questionnaires were mailed to the study's main researchers. The clinician survey was administered via email to 1179 healthcare providers who were registered members of the Hellenic Diabetes Association, the Cyprus Society For The Study Of Diabetes (ETDICY Society) or a network of general practitioners across Greece.

\section{Quantitative Data Analysis}

We used descriptive statistics to summarize the demographic characteristics of patients and clinicians and their responses to the 3 survey questions. We compared patients' preferences with those of clinicians by calculating odds ratios (ORs) with 95\% confidence intervals (CIs). We conducted multiple regression analyses to examine the association between patients' and clinicians' choices (dependent variables) with demographic characteristics (independent variables). Statistical analyses were undertaken in SPSS v25.0.

\section{RESULTS}

\section{Focus Group Study Participants}

Initially, 68 patients were identified from 4 clinics and volunteered to participate in the focus group study. Of these, 35 patients either could not be re-contacted by phone to affirm their participation or did not show up in their scheduled focus group session. The remaining 33 patients took part in 6 focus group sessions. Sessions lasted between 60 and $90 \mathrm{~min}$, while the number of sessions for each group was not predefined, but was dependent on the number of participants and the degree of interaction between members of each group. Three sessions were done with patients who had T2DM for less than 10 years (17 patients), and 3 sessions included patients with a duration of T2DM equal to or more than 10 years (16 patients). Two groups of patients participated in 4 sessions ( 2 sessions for each group), while 1 session took place for each of the remaining 2 groups of patients. The number of patients in each session was 7 or 8 in 4 sessions and 5 and 10 in 2 sessions. Most patients were male $(n=20)$, and their age ranged between 52 and 81 years (median $=63$ years, interquartile range $=58$ to 72 years). In general, the observer of the focus groups noted that group dynamics were positive and all participants contributed equally to the conversation in each session.

\section{Patients' Perspectives}

Emerging themes that were related to the aims of the mixedmethods study included long-term complications of T2DM, glycemic control, drug-related adverse events, impact of T2DM on daily life and routine, issues about insulin and other injectable therapies, and clinical parameters other than glucose control. We identified a total of 15 subthemes derived from the 
6 main themes. Exemplar quotes from patients supporting these themes and subthemes are presented in Appendix Text Box 3. Based on these (sub)themes, we identified the most prominent and clinically relevant antidiabetic medicationrelated outcomes (e.g., glycemic efficacy, incidence of hypoglycemia, or effect on individual macrovascular and microvascular endpoints) and other characteristics (e.g., drug cost or dosing scheme) to inform the answer options in survey questions 1 and 3, respectively (Text Box 1).

\section{Text Box 1 Patient survey questions}

Question 1
What is most important in a drug for diabetes?
Select the FIVE (5) most important for you:
1. Losing body weight
2. Preventing amputation of lower limbs and toes
3. Preventing a heart attack (myocardial infarction)
4. Preventing sexual dysfunction due to diabetes
5. Lowering blood glucose (glycated hemoglobin)
6. Preventing blinding and other eye complications of diabetes
7. Preventing a stroke
8. Preventing the development of kidney failure that requires dialysis
9. Lowering cholesterol and triglycerides
10. Not causing hypoglycemia
11. Lowering blood pressure
12. Helping you live more years
13. Other:
Question 2
Among your 5 selections which ONE (1) is your top choice?
Answer:
Question 3
If there were two drugs for diabetes with similar effects on the parameters mentioned above, which one
would you choose based on the following attributes?
Select the ONE (1) most important deciding factor for you:
1. The cheapest
2. The pill rather than the injection
3. The drug which does not require you to test your blood sugar with a finger prick every day
4. The drug which you have to take less frequently (for example once daily or even once a week)
5. Other:
(n)

\section{Survey Population}

A total of 656 patients with T2DM, recruited from 9 clinics, replied to the survey. We did not keep a log of all patients that were asked to participate; however, based on feedback from the participating clinics, the response rate of the patient survey was almost $100 \%$. Of 1179 healthcare providers who received an email with a link to the survey, $395(33.6 \%)$ responded. Of these, $363(30.8 \%)$ clinician responses (215 internists, 118 general practitioners, and 30 endocrinologists) were included in the analysis. The 32 responses that were excluded were from non-clinicians (12 dieticians, 5 nurses, 3 pharmacists, and 2 health visitors) or from clinicians with a specialty other than internal medicine, endocrinology, or general medicine (10 responses, mostly from pediatricians). There were no missing data for the survey first question, while 80 patients and 43 clinicians did not respond to the second question. The third question was answered by all patients and was omitted by 5 clinicians. The demographic characteristics of patients and clinicians are presented in the Table 1 .
Table 1 Demographics of Patients and Clinicians Included in the Survey Analysis

\begin{tabular}{|c|c|}
\hline \multicolumn{2}{|l|}{ Patients $(n=656)$} \\
\hline Characteristic & No. $(\%)$ \\
\hline \multicolumn{2}{|l|}{ Age (years) } \\
\hline$\leq 49$ & $50(7.8)$ \\
\hline$\overline{5} 0-64$ & $227(35.5)$ \\
\hline $65-79$ & $311(48.6)$ \\
\hline$\geq 80$ & $52(8.1)$ \\
\hline Female & $304(46.6)$ \\
\hline \multicolumn{2}{|l|}{ Duration of diabetes (years) } \\
\hline$<5$ & $128(19.9)$ \\
\hline $5-9$ & $154(23.9)$ \\
\hline$\geq 10$ & $362(56.2)$ \\
\hline \multicolumn{2}{|l|}{ No. of medications except insulin } \\
\hline $0-1$ & $309(49.7)$ \\
\hline 2 & $203(32.7)$ \\
\hline$\geq 3$ & 109 (17.6) \\
\hline On insulin therapy & $266(40.7)$ \\
\hline \multicolumn{2}{|l|}{ Level of diabetes care } \\
\hline Primary (3 practices) & $107(16.3)$ \\
\hline Secondary (6 practices) & $549(83.7)$ \\
\hline \multicolumn{2}{|l|}{ Location } \\
\hline Urban (8 practices) & $617(94.0)$ \\
\hline Rural (1 practice) & $39(6.0)$ \\
\hline \multicolumn{2}{|l|}{ Type of practice } \\
\hline Diabetes outpatient practice ( 7 practices) & $580(88.4)$ \\
\hline General practice ( 2 practices) & $76(11.6)$ \\
\hline \multicolumn{2}{|l|}{ Clinicians $(n=363)$} \\
\hline Characteristic & No. $(\%)$ \\
\hline Female & $146(40.4)$ \\
\hline \multicolumn{2}{|l|}{ Specialty } \\
\hline Internists & $215(59.2)$ \\
\hline General practitioners & $118(32.5)$ \\
\hline Endocrinologists & $30(8.3)$ \\
\hline \multicolumn{2}{|l|}{ Internists and self-reported training in diabetes } \\
\hline Certified training & $115(54.2)$ \\
\hline Postgraduate education & $59(27.8)$ \\
\hline None & 38 (17.9) \\
\hline \multicolumn{2}{|c|}{ General practitioners and self-reported training in diabetes } \\
\hline Certified training & $0(0.0)$ \\
\hline Postgraduate education & $43(37.1)$ \\
\hline None & $73(62.9)$ \\
\hline
\end{tabular}

\section{Preferences on Diabetes-Related Outcomes}

The distribution of patients' and clinicians' answers, when asked to choose the 5 most important among 12 possible treatment outcomes (survey question 1), is shown in Appendix Fig. 1. The 5 most frequently endorsed outcomes for patients were prevention of diabetic eye disease, prevention of myocardial infarction, $\mathrm{HbA} 1 \mathrm{c}$ reduction, and stroke or amputation prevention. The 5 most frequently endorsed outcomes for clinicians were low risk for hypoglycemia, reduction of $\mathrm{HbA1c}$, mortality reduction, prevention of myocardial infarction, and reduction in body weight. The ORs comparing patients' choices with those of clinicians are shown in Fig. 1. Patients were more likely than clinicians to rate prevention of amputation (OR 9.32; 95\% CI 6.51 to 13.35 ), diabetic eye disease $(6.16 ; 4.63$ to 8.21$)$, sexual dysfunction $(5.46 ; 2.71$ to $11.02)$, and stroke $(2.85 ; 2.17$ to 3.74$)$ as important, while clinicians were more likely than patients to choose low risk for hypoglycemia, and reduction of mortality, body weight, and HbAlc (Fig. 1).

The distribution of patients' and clinicians' answers when asked to report their top choice for the most important 


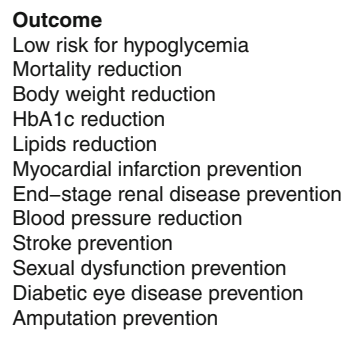

Outcome

ase prevention

Amputation prevention

$\begin{array}{cc}\text { Patients (n) } & \text { Clinicians (n) } \\ 206 & 290 \\ 196 & 255 \\ 249 & 220 \\ 414 & 285 \\ 67 & 34 \\ 436 & 232 \\ 340 & 172 \\ 74 & 24 \\ 363 & 110 \\ 80 & 9 \\ 450 & 95 \\ 356 & 41\end{array}$

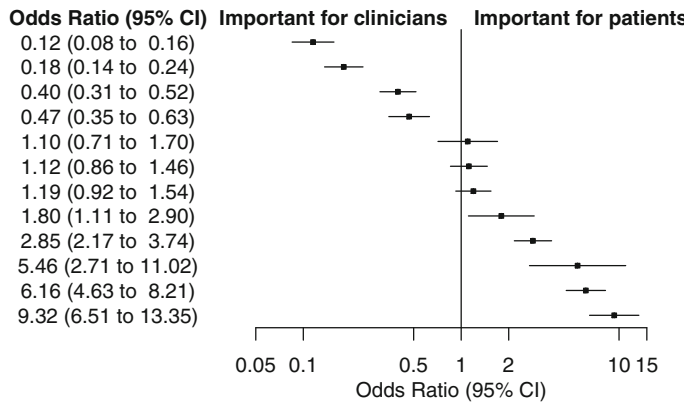

Figure 1 Patients' and clinicians' responses and odds ratios for the most frequently endorsed outcomes (survey question 1).

outcome (survey question 2) is shown in Appendix Fig. 2. The ORs comparing patients' responses with those of clinicians are shown in Fig. 2. Patients were more likely than clinicians to rate prevention of diabetic eye disease, amputation, stroke, chronic kidney disease, and myocardial infarction as the most important outcome, while clinicians were more concerned about reduction of all-cause mortality and $\mathrm{HbA} 1 \mathrm{c}$ and low risk for hypoglycemia (Fig. 2). In regression analyses, clinicians who reported having a certified training in diabetes were more likely to choose reduction of $\mathrm{HbAlc}$ as the most important outcome compared with those who reported not having received relevant training (Appendix Table 1).

\section{Preferences on Medication Attributes}

Less frequent administration was considered as the most important medication attribute both by patients and clinicians. The second most important attribute was oral (instead of injectable) administration for patients and drug cost for clinicians (Appendix Fig. 3). Relative comparisons showed that patients were less concerned than clinicians about drug cost (OR 0.16;95\% CI 0.11 to 0.23 ), but more concerned about route of administration $(2.56 ; 1.85$ to 3.54$)$ and need for less frequent glucose self-monitoring (1.64; 1.14 to 2.37) (Fig. 3). In regression analyses, patients taking less than 2 anti- diabetic drugs were more likely to consider frequency of drug administration as an important attribute compared with patients taking at least 2 medications (Appendix Table 2).

\section{DISCUSSION}

\section{Summary of Findings}

Focus group data from 33 patients with T2DM suggested that patients fear most the development of longterm complications, and in particular blindness, amputation, and end-stage renal disease. Notably, some patients explicitly reported that they valued quality of life more than overall survival, in the sense that they would prefer to live fewer years without debilitating symptoms, instead of living a longer life while being bedridden or constantly requiring the help of others. The importance of maintaining stable glucose control was also prominent among focus group participants, while concerns about drug-related adverse effects were mainly focused on hypoglycemia. Furthermore, many patients addressed the issue of polypharmacy, while some expressed their worries about treatment with insulin or other injectable therapies.
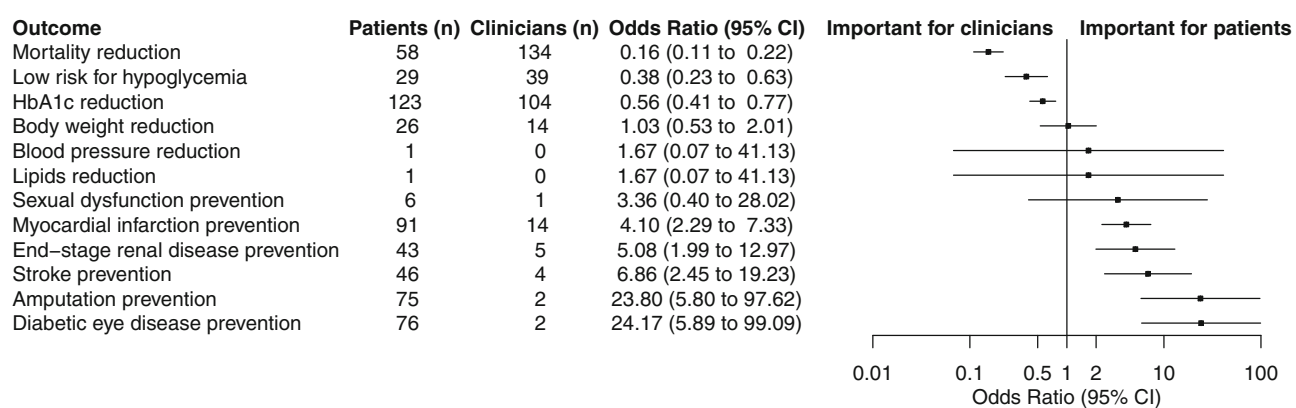

Figure 2 Patients' and clinicians' responses and odds ratios for the most important outcome (survey question 2). 


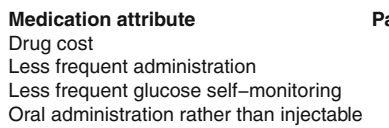

$\begin{array}{ccc} & & \\ & \text { Patients (n) } & \text { Clinicians (n) } \\ & 45 & 114 \\ & 241 & 129 \\ & 128 & 46 \\ & 128 & \\ & 217 & 58\end{array}$

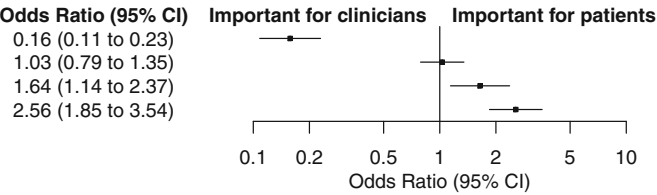

Figure 3 Patients' and clinicians' responses and odds ratios for the most important medication attribute (survey question 3).

Our findings from the survey study suggest that clinicians place more value on reduction of mortality, $\mathrm{HbAlc}$, and risk for hypoglycemia compared with other potential effects of antidiabetic medications. Patients also considered glycemic efficacy important, but they were also concerned about prevention of vascular complications including eye complications, amputation, myocardial infarction, stroke, and chronic kidney disease. Relative effect estimates comparing patients' and clinicians' preferences indicated that patients consider T2DM complications more important, as opposed to glycemic control, hypoglycemia, or all-cause mortality. Comparisons between patients' and clinicians' preferences about medication attributes showed that patients placed more importance on issues related to route of administration and frequency of self-glucose monitoring, while clinicians were more concerned about drug cost.

\section{Comparison with Other Studies}

A 2012 systematic review of 10 cross-sectional studies assessing patient preferences for non-insulin medications for $\mathrm{T} 2 \mathrm{DM}^{11}$ and three recent pertinent studies ${ }^{32-34}$ concluded that glycemic efficacy, effect on body weight, risk for hypoglycemia, and incidence of gastrointestinal side effects were the most influential attributes driving patients' preferences. In our study, patients with T2DM did consider glycemic efficacy as an important outcome, but, as opposed to the other studies, they placed greater relative value on prevention of eye complications, amputation, and myocardial infarction compared with reduction in body weight and low hypoglycemic risk. However, findings are not directly comparable between our study and previous studies, due to differences in methodological design ${ }^{11,34}$ and in choice of treatment outcomes and attributes assessed. ${ }^{32,} 33$ On the other hand, consistent with our findings, a survey study in more than 2000 patients with type 2 or type 1 diabetes in the USA concluded that patients prefer that diabetes trials focus more on patient-important outcomes, such as blindness, end-stage renal disease, and cardiovascular endpoints, rather than $\mathrm{HbAlc}$ and other surrogate outcomes. $^{35}$

Few studies have assessed both patients' and clinicians' preferences regarding treatment of T2DM. ${ }^{12,}{ }^{13,}{ }^{36}$ Even though these studies implemented a different design and did not address the same research question with our study, they also demonstrated that there is a disparity between patients' and clinicians' preferences. Of note, this discordance between patient and clinician perceptions has also been made evident in health conditions, other than T2DM. ${ }^{3,}{ }^{37}$ In our study, we highlighted this disagreement by calculating odds ratios comparing patients' and clinicians' responses. Moreover, although some previous studies have included a qualitative component for informing a survey development, they focused almost exclusively on the quantitative part when presenting their findings. ${ }^{12,}{ }^{32}$ Instead, we implemented a sequential, mixedmethods design to incorporate both qualitative and quantitative findings. ${ }^{16}$

\section{Study Limitations}

Even though both the patient and the clinician survey used identical questions, interpretation of some items might not have been the same, due to differences between patients' and clinicians' background knowledge on research evidence for T2DM. For example, knowledge of recent clinical trial findings suggesting a potential favorable effect of newer antidiabetic medications on mortality outcomes ${ }^{38}$ may have led more clinicians to choose all-cause mortality as an important outcome, as opposed to patients who may not be aware of such information. Moreover, due to the relatively small number of participating clinics $(n=9)$ in the survey study, patient preferences might not be representative of the views of the entire patient population with T2DM. In addition, only a small percentage of surveyed patients received care in a rural practice, and thus, this study cannot capture any potential differences in patients' preferences between urban and rural areas. Generalizability of our results could also be attenuated, because our study was conducted exclusively within the Greek healthcare setting. In particular, patients in our survey did not seem to consider drug cost as an important deciding factor, probably because patient co-payment in medications is relatively low in Greece, which might not be the case in other countries with different reimbursement systems. However, except for medication cost, there is no specific reason to believe that patients' and clinicians' preferences on other attributes would significantly differ from those in other European countries or in the USA, since there are no important country-specific differences between these regions and Greece in terms of available medication options for T2DM. ${ }^{9}$ Finally, we conducted focus groups solely with patients and not with clinicians. Conducting focus groups 
also with clinicians could probably elicit additional information to inform the survey content in terms of diabetesrelated outcomes and medication attributes.

\section{CONCLUSIONS}

Patients and clinicians may differ in their perceptions of the relative importance of various treatment outcomes and medication characteristics for T2DM, with patients being more concerned about parameters that can directly affect their quality of life. Such patient concerns and preferences should be discussed during the clinical encounter when deciding on an optimal therapeutic plan and should also guide the research agenda in T2DM.

Acknowledgments: We would like to thank the following for their contribution to this study: The Executive Committee of the Hellenic Diabetes Association for providing access and permission to distribute the clinician survey to its listed members. The Executive Committee of the Cyprus Society For The Study Of Diabetes (ETDICY Society) for providing access and permission to distribute the clinician survey to its listed members. Professor Benos A. and Assistant Professor Smyrnakis E. at the Laboratory of Primary Health Care, General Practice and Health Services Research, Aristotle University of Thessaloniki, for providing access and permission to distribute the clinician survey to a network of general practitioners across Greece. Professor Tentolouris N., Professor Papazoglou D., and Drs Mparmpa E. and Aggeloudi E. for assisting with patient recruitment. Dr. Malandris $K$. and Ms. Sachanidou Z. for assisting with data collection in the survey study. Ms. Papaggeli M. for assisting with data collection in the focus group study.

Corresponding Author: Thomas Karagiannis, MD, MSc, PhD; Clinical Research and Evidence-Based Medicine Unit, Second Medical DepartmentAristotle University of Thessaloniki, Thessaloniki, Greece (e-mail: tkaragian@auth.gr).

Author Contributions Dr. Karagiannis T. had full access to all the data in the study and takes responsibility for the integrity of data and the accuracy of the data analysis

Study concept and design: Karagiannis T, Bekiari E, Tsapas A, Liakos A Acquisition of data: Karagiannis T, Toumpalidou M, Kitsios K, Dimitriadis G. Papanas N. Bargiota A, Avramidis I, Katsoula A Tentolouris A, Chatziadamidou T, Giannakopoulos S, Alexiadis S, Kotsa K

Analysis of focus group study data: Karagiannis T, Toumpalidou M, Tsapas A, Bekiari E

Analysis of survey study data: Karagiannis T, Avgerinos I, Liakos A Interpretation of data: Karagiannis T, Avgerinos I, Toumpalidou M, Bekiari E, Liakos A, Tsapas A

Drafting of the manuscript: Karagiannis T, Avgerinos I

Critical revision of the manuscript: all authors

Obtained funding: Tsapas A

Supervision: Karagiannis T, Tsapas A, Bekiari E

Funding Information This study was supported by the European Foundation for the Study of Diabetes (EFSD) PAtient-Centred Treatment to support a holistic approach towards type 2 diabetes (PACT) Programme supported by AstraZeneca.

\section{Compliance with Ethical Standards:}

Conflict of Interest: Dr. Karagiannis T, Ms. Toumpalidou M, Dr. Tsapas A., and Dr. Bekiari E. have received payment for the submitted work through a grant by the European Foundation for the Study of Diabetes (EFSD) PAtient-Centred Treatment to support a holistic approach towards type 2 diabetes (PACT) Programme supported by AstraZeneca.

The remaining authors report no potential conflicts of interest relevant to this article.

Ethical Approval: This study was approved by the Bioethics Committee of the Medical School of the Aristotle University of Thessaloniki (No. 8/22.2.2017).

Disclaimer: The funder had no role in the design and conduct of the study, interpretation of the data, or preparation of the manuscript.

\section{REFERENCES}

1. Captieux M, Pearce G, Parke HL, et al. Supported self-management for people with type 2 diabetes: a meta-review of quantitative systematic reviews. BMJ open. 2018; 8:e024262.

2. Russell-Jones D, Dauchy A, Delgado E, et al. Take Control: A randomized trial evaluating the efficacy and safety of self- versus physician-managed titration of insulin glargine $300 \mathrm{U} / \mathrm{mL}$ in patients with uncontrolled type 2 diabetes. Diabetes, obesity \& metabolism. 2019; 21:1615-1624.

3. Muhlbacher AC, Juhnke C. Patient preferences versus physicians' judgement: does it make a difference in healthcare decision making? Applied health economics and health policy. 2013; 11:163-180.

4. Greene J, Hibbard JH, Sacks R, Overton V, Parrotta CD. When patient activation levels change, health outcomes and costs change, too. Health affairs. 2015; 34:431-437.

5. Adams RJ. Improving health outcomes with better patient understanding and education. Risk management and healthcare policy. 2010; 3:61-72.

6. Gagliardi AR, Legare F, Brouwers MC, Webster F, Badley E, Straus S. Patient-mediated knowledge translation (PKT) interventions for clinical encounters: a systematic review. Implementation science : IS. 2016; $11: 26$.

7. Sackett DL, Rosenberg WM, Gray JA, Haynes RB, Richardson WS. Evidence based medicine: what it is and what it isn't. Bmj. 1996; 312:71-72.

8. Inzucchi SE, Bergenstal RM, Buse JB, et al. Management of hyperglycaemia in type 2 diabetes: a patient-centered approach. Position statement of the American Diabetes Association (ADA) and the European Association for the Study of Diabetes (EASD). Diabetologia. 2012; 55:1577-1596.

9. Davies MJ, D'Alessio DA, Fradkin J, et al. Management of Hyperglycemia in Type 2 Diabetes, 2018. A Consensus Report by the American Diabetes Association (ADA) and the European Association for the Study of Diabetes (EASD). Diabetes care. 2018; 41:2669-2701.

10. Joy SM, Little E, Maruthur NM, Purnell TS, Bridges JF. Patient preferences for the treatment of type 2 diabetes: a scoping review. PharmacoEconomics. 2013; 31:877-892.

11. Purnell TS, Joy S, Little E, Bridges JF, Maruthur N. Patient preferences for noninsulin diabetes medications: a systematic review. Diabetes care. 2014; 37:2055-2062.

12. Porzsolt F, Clouth J, Deutschmann M, Hippler HJ. Preferences of diabetes patients and physicians: a feasibility study to identify the key indicators for appraisal of health care values. Health and quality of life outcomes. 2010; 8:125.

13. Morillas C, Feliciano R, Catalina PF, et al. Patients' and physicians' preferences for type 2 diabetes mellitus treatments in Spain and Portugal: a discrete choice experiment. Patient preference and adherence. 2015; 9:1443-1458.

14. Campbell DJ, Tam-Tham H, Dhaliwal KK, et al. Use of Mixed Methods Research in Research on Coronary Artery Disease, Diabetes Mellitus, and Hypertension: A Scoping Review. Circulation Cardiovascular quality and outcomes. 2017; 10.

15. Lopez JM, Katic BJ, Fitz-Randolph M, Jackson RA, Chow W, Mullins CD. Understanding preferences for type 2 diabetes mellitus selfmanagement support through a patient-centered approach: a 2-phase mixed-methods study. BMC endocrine disorders. 2016; 16:41. 
16. Fetters MD, Curry LA, Creswell JW. Achieving integration in mixed methods designs-principles and practices. Health services research. 2013; 48:2134-2156.

17. Tong A, Sainsbury P, Craig J. Consolidated criteria for reporting qualitative research (COREQ): a 32-item checklist for interviews and focus groups. International journal for quality in health care : journal of the International Society for Quality in Health Care. 2007; 19:349-357.

18. von Elm E, Altman DG, Egger M, et al. Strengthening the Reporting of Observational Studies in Epidemiology (STROBE) statement: guidelines for reporting observational studies. Bmj. 2007; 335:806-808.

19. Tausch AP, Menold N. Methodological Aspects of Focus Groups in Health Research: Results of Qualitative Interviews With Focus Group Moderators. Global qualitative nursing research. 2016; 3:2333393616630466.

20. Carolan M, Holman J, Ferrari M. Experiences of diabetes self-management: a focus group study among Australians with type 2 diabetes. Journal of clinical nursing. 2015; 24:1011-1023.

21. Herre AJ, Graue M, Kolltveit BC, Gjengedal E. Experience of knowledge and skills that are essential in self-managing a chronic condition - a focus group study among people with type 2 diabetes. Scandinavian journal of caring sciences. 2016; 30:382-390.

22. Trinkley KE, Malone DC, Nelson JA, Saseen JJ. Prescribing attitudes, behaviors and opinions regarding metformin for patients with diabetes: a focus group study. Therapeutic advances in chronic disease. 2016; 7:220-228.

23. Palinkas LA, Horwitz SM, Green CA, Wisdom JP, Duan N, Hoagwood K. Purposeful Sampling for Qualitative Data Collection and Analysis in Mixed Method Implementation Research. Administration and policy in mental health. 2015; 42:533-544.

24. Tang KC, Davis A. Critical factors in the determination of focus group size. Family practice. $1995 ; 12: 474-475$.

25. Huang ES, Laiteerapong N, Liu JY, John PM, Moffet HH, Karter AJ. Rates of complications and mortality in older patients with diabetes mellitus: the diabetes and aging study. JAMA internal medicine. 2014; 174:251-258.

26. Saunders B, Sim J, Kingstone T, et al. Saturation in qualitative research: exploring its conceptualization and operationalization. Quality \& quantity. 2018; 52:1893-1907.

27. Castleberry A, Nolen A. Thematic analysis of qualitative research data: Is it as easy as it sounds? Currents in pharmacy teaching \& learning. 2018; 10:807-815.

28. Noble H, Mitchell G. What is grounded theory? Evidence-based nursing. 2016; 19:34-35.
29. Harris PA, Taylor R, Thielke R, Payne J, Gonzalez N, Conde JG. Research electronic data capture (REDCap)-a metadata-driven methodology and workflow process for providing translational research informatics support. Journal of biomedical informatics. 2009; 42:377-381.

30. Liatis S, Dafoulas GE, Kani C, et al. The prevalence and treatment patterns of diabetes in the Greek population based on real-world data from the nation-wide prescription database. Diabetes research and clinical practice. 2016; 118:162-167.

31. The Hellenic Statistical Authority. Available at: http://www.statistics. gr/en/statistics/-/publication/SHE09/2016?zX=w5ygp6ix4j75 . Last accessed October 10, 2019.

32. Flood EM, Bell KF, de la Cruz MC, Ginchereau-Sowell FM. Patient preferences for diabetes treatment attributes and drug classes. Current medical research and opinion. 2017; 33:261-268.

33. Gelhorn HL, Stringer SM, Brooks A, et al. Preferences for medication attributes among patients with type 2 diabetes mellitus in the UK. Diabetes, obesity \& metabolism. 2013; 15:802-809.

34. Mansfield C, Sikirica MV, Pugh A, et al. Patient Preferences for Attributes of Type 2 Diabetes Mellitus Medications in Germany and Spain: An Online Discrete-Choice Experiment Survey. Diabetes therapy : research, treatment and education of diabetes and related disorders. 2017; 8:1365-1378.

35. Murad MH, Shah ND, Van Houten HK, et al. Individuals with diabetes preferred that future trials use patient-important outcomes and provide pragmatic inferences. Journal of clinical epidemiology. 2011; 64:743-748.

36. Chin MH, Drum ML, Jin L, Shook ME, Huang ES, Meltzer Do. Variation in treatment preferences and care goals among older patients with diabetes and their physicians. Medical care. 2008; 46:275-286.

37. Rothwell PM, McDowell Z, Wong CK, Dorman PJ. Doctors and patients don't agree: cross sectional study of patients' and doctors' perceptions and assessments of disability in multiple sclerosis. Bmj. 1997; 314:1580-1583.

38. Zhuang XD, He X, Yang DY, et al. Comparative cardiovascular outcomes in the era of novel anti-diabetic agents: a comprehensive network metaanalysis of 166,371 participants from 170 randomized controlled trials. Cardiovascular diabetology. 2018; 17:79.

Publisher's Note Springer Nature remains neutral with regard to jurisdictional claims in published maps and institutional affiliations. 